Trauma Berufskrankh $2005 \cdot 7$ [Suppl 2]: S340-S344 DOI 10.1007/s10039-004-0981-7

Online publiziert: 10. Dezember 2004

(c) Springer Medizin Verlag 2004
M. Arand - L. Kinzl

Abteilung für Unfallchirurgie, Hand- und Wiederherstellungschirurgie, Universität UIm

\section{Pathomechanik der thorakolumbalen Verletzung}

\section{Was heißt eigentlich Instabilität?}

irbelsäulenverletzungen des thorakolumbalen Übergangs haben in den vergangenen Jahrzehnten in der Bundesrepublik Deutschland durch Veränderungen im Freizeitverhalten sowie ein erhöhtes Verkehrsaufkommen kontinuierlich zugenommen. Voraussetzung für eine adäquate Therapieentscheidung auf Grundlage einer nachvollziehbaren und reproduzierbaren Indikationsstellung ist eine umfassende klinische, neurologische sowie radiologische Verletzungsdiagnostik, aus welcher die Pathomechanik der Verletzung und die Instabilität zu eruieren sind. Die Klassifikation der Verletzung mit einer systematischen Einteilung verschiedener Verletzungsgruppen ergibt sich aus der segmentalen und ossären Radiomorphologie und erlaubt eine Standardisierung indikatorischer und therapeutischer Faktoren.

\section{Anatomische Grundlagen}

Im Bereich des zervikalen und lumbalen Abschnitts bestehen jeweils lordotische Ausschwingungen, die thorakolumbale Übergangsregion ist geprägt vom Übergang der thorakalen Kyphose in die lumbale Lordose. Adaptiert an die mechanischen Ansprüche nimmt die Größe der Wirbelkörper von der HWS zur LWS deutlich zu, gleiches gilt für die Größen- und Massenverhältnisse der Pedikel sowie der Facetten der kleinen Wirbelgelenke.

Das relative Platzangebot des Spinalkanals für die neurogenen Strukturen ist in Relation zu deren Platzbedarf groß, volu- menanteilig werden $<50 \%$ des Platzangebots beansprucht. Die einzige, wenn auch leichte Unterschreitung dieser Ratio findet sich im Bereich der BWS zwischen Th4 und Thy.

Im Bereich des thorakolumbalen Übergangs bzw. etwas distal davon befindet sich der Übergang des Rückenmarks in die Cauda equina.

Die Blutversorgung des Rückenmarks im thorakolumbalen Übergang erfolgt vorwiegend aus Ästen der Segmentgefäße, die aus der A. spinalis anterior gespeist werden. In diesem Bereich gibt es ausreichend Umgehungskreisläufe. In der Region Th5 und Th7 dagegen liegt eine arterielle Endstreckenversorgung vor.

Die Bandscheiben bestehen aus einem weichen, gallertigen Kern (Nucleus pulposus) und einem harten Faserring (Anulus fibrosus). Die Ernährung beider Systeme wird durch Diffusion aufrechterhalten. Die zirkulär und laminarförmig um den Nucleus pulposus angeordneten elastischen Fasersysteme des Anulus gewährleisten durch den Einstrahlungswinkel der Fibrillen von $30^{\circ}$ in die Grund- und Deckplatten eine stabile und tragfähige Verbindung des Anschlusswirbels sowie die $\mathrm{Si}$ cherung des Nukleus.

Die Gelenkfacetten weisen im Bereich des thorakolumbalen Übergangs zwischen BWS und LWS eine wesentliche Veränderung der Ausrichtung auf. Während die Gelenkebene im Bereich der BWS in der Frontalebene gekippt ist, ist sie im Bereich der LWS in der Sagittalebene ausgerichtet.
Aus der bereits sagittalen Gelenkstellung resultiert im Bereich des thorakolumbalen Übergangs eine Limitation der Rotation durch die ossären Bestandteile der Facettengelenke, funktionell weist dieser $\mathrm{Ab}$ schnitt deshalb die geringste segmentale Beweglichkeit für Rotation im Bereich der gesamten Wirbelsäule auf. Die segmentale Beweglichkeit für Flexion und Extension sowie Lateralflexion sind durchschnittlich und ligamentär limitiert.

\section{Mechanische Grundlagen}

Das Funktionsprinzip und die Lastverteilung der Wirbelsäule sind segmental abhängig vom Ausmaß des darüber abhängigen Körpergewichts und dessen mechanischer Voraussetzungen. Die Funktionsweise ist der eines Lastkrans vergleichbar [1]. Während die ventralen Abschnitte der Wirbelsäule (Wirbelkörper, Bandscheiben) wie der Krankörper den Großteil der axialen Lasten zu übernehmen haben, obliegt es den dorsalen Elementen der Wirbelsäule (dorsale Bandkomplexe, Muskulatur), korrespondierend dem Zugseil die auftretenden Zugkräfte zu egalisieren. Zur Erklärung der statischen Lastverhältnisse dient das 3-Säulen-Modell nach Louis [4]. Demnach ist die vordere Säule durch die Wirbelkörper und die Bandscheiben, die hintere Säule durch die beiden symmetrisch angelegten Facettenregionen repräsentiert.

Bei Frakturen des Wirbelkörpers kommt es durch die segmentale kyphotische Deformation zu einer Ventralisie- 
rung der kranial des Läsionsorts liegenden Wirbelsäule. Daraus resultiert eine Vorverlagerung des Schwerpunkts der kranial der Fehlstellung liegenden Körpermasse (- Abb. 1), sodass aus biomechanischer Sicht eine zusätzliche Fehlbelastung des bereits geschädigten Areals entsteht, welche zu einer wiederum ungünstigen Beeinflussung der Fraktur führt.

Die kompressive Belastbarkeit des Wirbelkörpers nimmt unter Voraussetzung einer intakten Strukturierung und Vitalität des Knochens von kranial nach kaudal zu. Im Bereich des thorakolumbalen Übergangs bestehen axiale Belastungsfähigkeiten des Wirbelkörpers in seinem Gesamtflächenquerschnitt von 4000-600o Nm [6]. Eine Abnahme dieser kompressiven Belastungsfähigkeit ist z. B. durch osteoporotische Erkrankungen auf bis zu 10\% der Norm gegeben.

Die Verteilung der Widerstandsfähigkeit eines thorakolumbalen Wirbels im Rahmen einer axialen Belastung (- Abb. 2) zeigt eine hohe axiale Belastbarkeit insbesondere im Bereich der Facetten, der Pedikelregion und der hinteren Korpusregion sowie eine relativ geringe kompressive Belastbarkeit im Bereich der zentralen Anteile der Deckplatte mit wieder leicht ansteigender Tendenz in Richtung Vorderkante. Wird die axiale Widerstandsfähigkeit der Wirbelkörperspongiosa nach Entfernung der kortikalen Lamelle sowie der Grund- und Deckplatten gemessen, zeigt sich in Abweichung zu den intakten Verhältnissen eine erhöhte kompressive Belastbarkeit im Zentrum der Spongiosa mit Verringerung dieser Widerstandskraft in den peripheren Anteilen [3].

Die Reißfestigkeit der Bänder (• Tabelle 1) im Bereich des thorakolumbalen Übergangs [7] ist am höchsten für das vordere Längsband gegenüber Extension. Das Zentrum der Rotation bei Extension des kranialen Wirbels liegt hierbei im Bereich der dorsalen kaudalen Wirbelkörperecke in Projektion auf die hinteren Anteile der Bandscheibe (• Abb. 3). Die Reißfestigkeiten für das Lig. supraspinosum, für die Kapselbänder und das Lig. flavum sind aufgrund der mehrfachen Bandbesetzung bei weiter vorne liegendem Drehpunkt in Flexion als widerstandsfähiger gegenüber Rupturen zu beurteilen.

Trauma Berufskrankh $2005 \cdot 7$ [Suppl 2]: S340-S344

DOI 10.1007/s10039-004-0981-7

(c) Springer Medizin Verlag 2004

M. Arand · L. Kinzl

\section{Pathomechanik der thorakolumbalen Verletzung. Was heißt eigentlich Instabilität?}

\section{Zusammenfassung}

Verletzungen des thorakolumbalen Übergangs resultieren im Wesentlichen aus Kompression, Flexion/Distraktion und Torsion. Kompression und Torsion führen überwiegend zu knöchernen Verletzungen, Flexion, Distraktion und Seitneigung v. a. zu ligamentären Instabilitäten. Der Terminus physikalische Instabilität der Wirbelsäule ist aus klinisch-medizinischer Sicht für die Zustandsbeschreibung von thorakolumbalen Verletzungen als relativ einzustufen. Als allgemeiner Instabilitätsbegriff hat sich folgende Definition als vorteilhaft erwiesen: Verlust der Wirbelsäule, ihre physiologischen Aufgaben wie Transfer von Lasten und Momenten, Bewegungsmöglichkeit sowie Schutz der neurogenen Struktu- ren zu übernehmen. Eine Quantifizierung der Instabilität einer einzelnen Verletzung ist nicht praktikabel, radiologische Kriterien dienen zur Präzisierung der korporalen oder segmentalen Instabilität. Grundlage der Bewertung sind radiomorphologische Kriterien. Zu ihrer vollständiger Erfassung sind Nativaufnahmen in 2 Ebenen und eine Computertomographie abzuleisten, teilweise sind funktionsdiagnostische Maßnahmen erforderlich.

\section{Schlüsselwörter}

Thorakolumbale Verletzung . Instabilitätsbegriff · Radiologische Kriterien · Radiomorphologische Kriterien .

Funktionsdiagnostische Maßnahmen

\section{Pathomechanisms of thoracolumbar injury. What does instability actually mean?}

\begin{abstract}
Injuries of the thoracolumbar junction basically result from three mechanisms: compression, flexion/distraction and torsion. Compression and torsion lead mainly to bony injury patterns and flexion, distraction and lateral bending, mainly to ligamentous instabilities. The meaning of "instability" is clear, but when applied to the clinical status of thoracolumbar fractures instability can only be relative. In general, it seems helpful to define "instability" as loss of the ability of the spine to maintain its physiological tasks, such as the transfer of loads and moments, movement, and protection of neurological structures. Quantification of the instability
\end{abstract}

of an individual thoracolumbar injury is not possible, radiologic criteria of instability allow more precise identification of bony and ligamentous instability. Radiomorphologic data are the basis of the evaluation. Native $X$-rays (a-p and lateral views) and computed tomography are necessary for ascertainment of all these parameters, and in some cases functional $\mathrm{X}$-rays are essential.

\section{Keywords}

Injury at the thoracolumbar junction . Meaning of instability - Radiological criteria . Radiomorphological criteria .

Functional diagnostic tests 


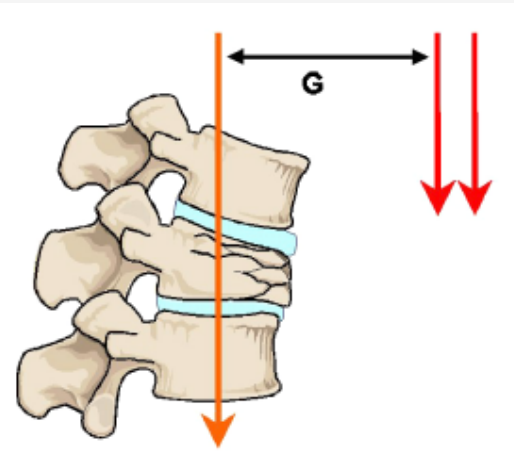

Abb. $1 \Delta$ Schwerpunktverlagerung durch traumatische angulierende Wirbelkörperdeformationen, Ventralisierung der proximal abhängigen Wirbelsäule mit Verstärkung der kyphosierenden Belastung auf das verletzte Segment

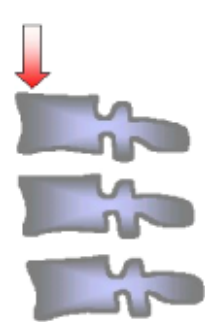

A1
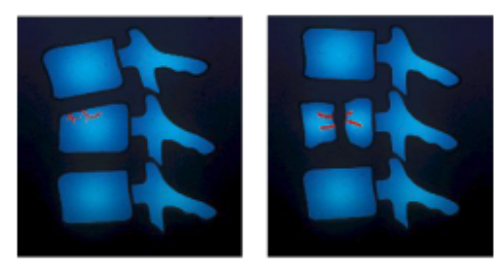

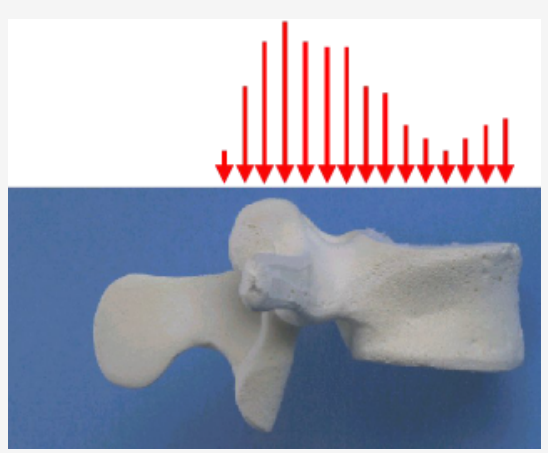

Abb. $2 \Delta$ Verteilung der axialen Widerstandsfähigkeit des intakten Wirbels

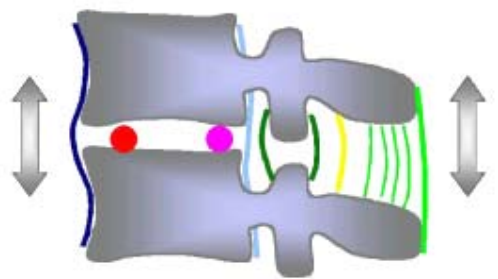

Abb. $3 \triangle$ Zentrum der Extensions- (rosa) und Flexionsrotation (rot) sowie Bandkonstellation dazu: Lig. longitudinale anterius (dunkelblau), Lig. longitudinale posterius (hellblau), Kapselbänder (dunkelgrün), Lig. flavum (gelb), intra-/supraspinöse Bänder (hellgrün)

Abb. $4 \Delta$ Pathomechanismus und Morphologie der Kompressionsverletzungen (Typ $A$ )

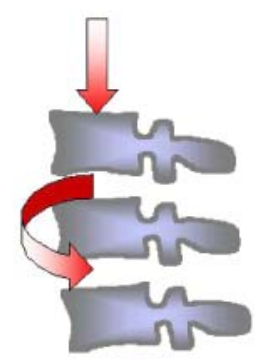

C1

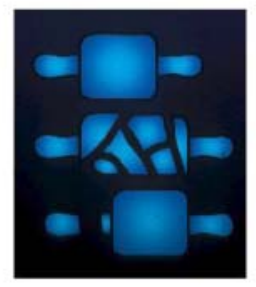

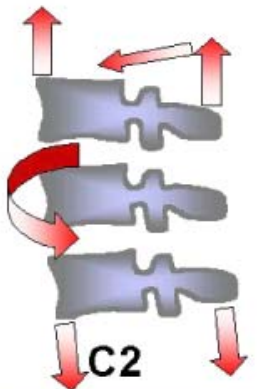

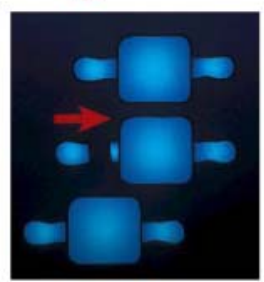

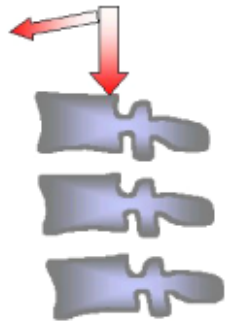

A3

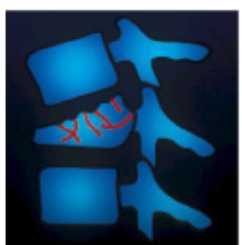

B1
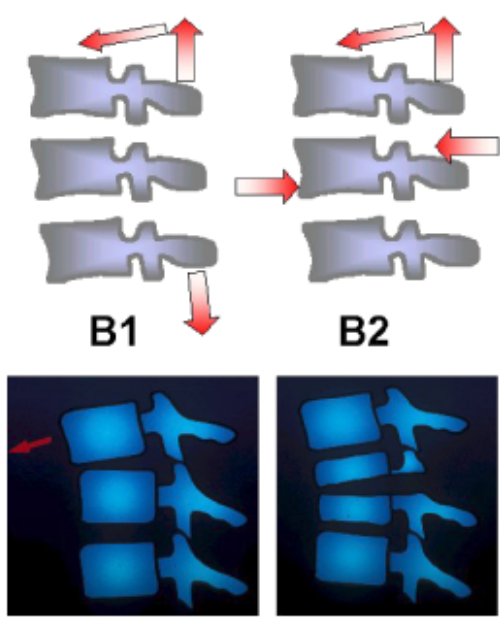

B2

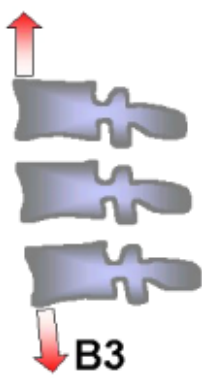

Abb.5 Pathomechanismus und Morphologie der Flexions/ -Distraktions- und Extensionsverletzungen (Typ B)

stabil

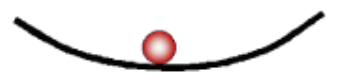

labil

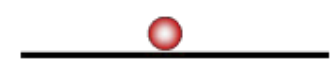

instabil

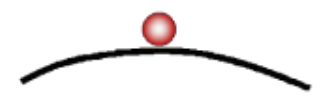

Abb. $7 \triangle$ Physikalische Definition der Begriffe Stabilität und Instabilität mit ihrer Zwischenform Labilität 


\section{Pathomechanik der Verletzung}

Das traumatische Übersteigen der Widerstandskraft (ossäre Bewegungslimitation) knöcherner Strukturen am thorakolumbalen Übergang führt zu Frakturen. Dies gilt unter Berücksichtigung der anatomischen Voraussetzung in erster Linie für die Torsionsbelastung und die Hyperkompression.

Werden die plastischen Dehnungseigenschaften der ligamentären Verbindungen überfordert (ligamentäre Bewegungslimitation), sind segmentale Translationen oder Distraktionen zu erwarten. Dies gilt am thorakolumbalen Übergang für Flexion und Extension sowie Seitneigung, die Inzidenz der Bandverletzungen ist in dieser Lokalisation jedoch niedrig.

Das Verletzungsausmaß an der thorakolumbalen Wirbelsäule ist abhängig vom

- Schädigungsmechanismus,

- den Grundvoraussetzungen des verletzten Organismus (Gewicht, Muskulatur sowie knöcherne und ligamentäre Festigkeit) sowie

- der Intensität der eingeleiteten Kraft bzw. des eingeleiteten Moments.

Aus der Pathomechanik des Unfalls resultiert ein Schädigungsmuster an der Wirbelsäule, welches eine Klassifikation erfordert. Die 1994 von Magerl et al. [5] vorgestellte AO-Klassifikation basiert auf pathomechanischen Grundlagen und beruht auf der AO-Unterteilung in 3 Verletzungstypen:

- Typ A: Kompressionsverletzung

- Typ B: Flexions-Distraktions- und Extensionsverletzungen

- Typ C: Torsionsverletzungen

Jeder Hauptgruppe der Verletzung sind 3 weitere Gruppen zugeteilt, die wiederum in weitere Untergruppen unterteilt sind. Sowohl innerhalb der Hauptgruppen von A nach $\mathrm{C}$ als auch in den Gruppen und Untergruppen von 1 nach 3 nimmt die Instabilität der Verletzung zu. Für die klinische Anwendung reicht die Verwendung der Hauptgruppen und Gruppen aus, für wissenschaftliche Fragestellungen ist eine Differenzierung der Verletzung bis in die Untergruppe sinnvoll.
Verletzungen vom Typ A. Bei den durch axiale Kompression hervorgerufenen Verletzungen ist in der Regel lediglich die vordere Säule betroffen ( $\bullet$ Abb. 4).

Bei den Impressions- oder Keilfrakturen (Typ A1) reicht eine moderate und ventral exzentrisch liegende axiale Kompressionsbelastung zur Frakturentstehung aus. Höhere und zentral liegende axiale Belastungen führen zum frontalen Spaltbruch (Kneifzangenbruch, Typ A2). Dorsal liegende axiale Belastungen mit zusätzlichem Flexionsmoment sind durch die typische Morphologie des inkompletten (Typ A3.1) oder kompletten (Typ A3.3) Berstungsbruchs mit Schädigung der Hinterkante gekennzeichnet.

Verletzungen vom Typ B. Bei den durch Flexion, Distraktion und Extension verursachten Verletzungen sind in der Regel alle 3 Säulen, die ventrale und die beiden dorsalen, betroffen ( Abb. 5). In Einzelfällen sind auch partielle Rupturen mit Beteiligung von 1 oder 2 Säulen denkbar.

Die ligamentäre Zerreißung (Typ B1) entsteht durch eine dorsale Distraktion bei simultaner Einleitung einer Flexion. Der ossären Zerreißung (Typ B2, „chance fracture") liegt eine dorsale Distraktion und gleichzeitige korporale Scherung zugrunde. Die im Bereich der thorakolumbalen Wirbelsäule seltene Hyperextension (Typ B3) entsteht durch ventrale Distraktion.

Verletzungen vom Typ C. Bei Torsionsverletzungen ( $\mathbf{A b b}$. 6) der thorakolumbalen Wirbelsäule sind generell alle 3 Säulen geschädigt.

Unter den Typ-C1-Verletzungen werden alle Torsionsverletzungen subsumiert, welche als zusätzliches Schädigungsmoment Kompression aufweisen (vom Rotationskeilbruch bis zum Rotationsberstungsbruch). Die Torsionsverletzungen vom Typ $\mathrm{C}_{2}$ entstehen durch eine zur Torsion hinzutretende Distraktion, es kommt einerseits vorwiegend zu ligamentären Läsionen wie Rotationssubluxationen oder Rotationsluxationen, aber auch zu vorwiegend knöchernen Schädigungen wie Rotationswirbelzerreißungen. Die Rotationsscherbrüche (Typ $\mathrm{C}_{3}$ ) bilden eine in sich inhomogene Gruppe an Verletzungen, die ätiopathologisch und morphologisch nicht brauchbar zu klassifizieren sind.
Tabelle 1

Reißfestigkeiten thorakolumbaler

Bänder

Band

Reißfestigkeit

[N]

\begin{tabular}{lr}
\hline Vorderes Längsband & $300-450$ \\
\hline Hinteres Längsband & $100-320$ \\
\hline Lig. flavum & $200-280$ \\
\hline Kapselbänder & $170-220$ \\
\hline Lig. intraspinosum & $80-120$ \\
\hline Lig. supraspinosum & $320-150$
\end{tabular}

Nach White u. Panjabi [7]

Tabelle 2

Biomechanische thorakolumbale

Instabilität, bei $\geq 5$ Punkten

Schädigung

Punkte

Schädigung „ventrale Elemente“ 2

Schädigung 2

"dorsaler Strukturen"

Rupturen kostovertebral $\quad 1$

Radiologische Kriterien

(4)

- Sagittale Dislokation $>2,5 \mathrm{~mm} \quad 2$

- Sagittale Angulation $>5^{\circ}$

2

Rückenmark- $/$

2

Cauda-equina-Läsion

Axiale Belastungsfähigkeit

reduziert

Modifiziert nach White u. Panjabi [8]

Tabelle 3

Radiologische Kriterien der

Instabilität der thorakolumbalen

Wirbelsäule

Wirbelkörper- $\quad 40-30 \%$

kompression

\begin{tabular}{lc} 
Segmentale Angulation & $5-20^{\circ}$ \\
\hline Segmentale Torsion & $30-10^{\circ}$ \\
\hline Segmentale Translation & $2,5-4,5 \mathrm{~mm}$ \\
\hline Laterale Aufklappbarkeit & $>2 \mathrm{~mm}$ \\
\hline Facettenüberlappung & $<50 \%$
\end{tabular}

\section{Instabilität}

Physikalisch. Die physikalische Definition der Instabilität ist eindeutig und klar in der Abgrenzung zur Stabilität (• Abb. 7). 
Definition. In der Zustandsbeschreibung thorakolumbaler Wirbelsäulenverletzungen lässt sich Instabilität nicht in jedem Fall eindeutig und trennscharf von Stabilität abgrenzen. Dies resultiert einerseits daraus, dass sich fließende Übergänge zwischen bereits instabilen und noch stabilen Verletzungen (innerhalb der Gruppe Typ A1) zeigen, andererseits finden sich in der Begriffsdefinition der Instabilität inhomogene Einflussfaktoren aus den Bereichen Biomechanik, Klinik, Neurologie und Radiologie. Als allgemeiner Begriff der Instabilität ist die folgende Definition praktikabel: Verlust der Wirbelsäule, ihre physiologischen Aufgaben wie Transfer von Lasten und Momenten, Bewegungsmöglichkeit sowie Schutz der neurogenen Strukturen zu übernehmen.

Biomechanisch. White u. Panjabi [8] definierten den Instabilitätsbegriff 1990 aus biomechanischer Sicht folgendermaßen:

\section{- „Loss of ability of the spine under physiologic loads to maintain its pattern of displacement so that there is no initial or additional neurologic deficit, no major deformity, and no incapacitating pain".}

Sie stellten ein Punktesystem zur Evaluation dieser Instabilität vor (• Tabelle 2), ab einer Punktesumme von $\geq_{5}$ Punkten wurde von einer relevanten Instabilität eines Bewegungssegments bzw. eines Knochenabschnitts der thorakolumbalen Wirbelsäule ausgegangen.

Klinisch. Blauth [2] stellte eine Definition der klinischen Instabilität für ausschließlich frische traumatische Läsionen vor. Unter Würdigung der Relativität des Instabilitätsbegriffs differenzierte er zwischen

- geringgradig instabilen Verletzungen (funktionelle Behandlung führt zu sicherer Ausheilung ohne neurologisches Defizit oder Deformation) und

- hochgradig instabilen Verletzungen (funktionelle Behandlung lässt schwere Fehlstellungen und neurologische Verschlechterung erwarten).

Neurologisch. Die neurologische Instabilität wurde bereits lange vor dem Beginn der operativen Therapie direkt einem segmentalen radikulären oder medullären Funktionsausfall und gleichzeitig nachgewiesener knöcherner oder ligamentärer Verletzung der thorakolumbalen Wirbelsäule zugeordnet. Unter Berücksichtigung der segmentalen Befundausprägung ist klinisch die Läsionshöhe zu verifizieren.

Radiologisch. Die Definition der radiologischen Instabilität ist dann problematisch, wenn nur geringe Deformationen oder Dislokationen vorliegen. Während des Traumas selbst können weitaus größere Schädigungen präsent gewesen sein welche durch Ruhebilder nicht zu erfassen sind. Eine weitere Diagnostik, zunächst im Schnittbildverfahren und ggf. weiter im Rahmen einer Funktionsdiagnostik (Durchleuchtung, Funktionsaufnahmen usw.), deckt diese versteckten Instabilitäten in der Regel auf. Die Beteiligung einer Wirbelkörperhinterkante alleine ist kein klassisches Instabilitätskriterium, wie dies häufig postuliert wird. Die relevanten radiologischen Kriterien sind

- Wirbelkörperkompression,

- segmentale Angulation,

- segmentale Torsion,

- segmentale Translation und

- laterale Aufklappbarkeit (• Tabelle 3).

Resümee. Zur umfassenden Anwendung der Definition der Instabilität auf eine thorakolumbale Verletzung sind biomechanische, klinische, neurologische und radiologische Instabilitätskriterien zu beachten. Die korrekte Analyse einer Läsion und damit eine korrekte Indikationsstellung zur spezifischen Therapie wird nur dann gelingen, wenn alle genannten Kriterien entsprechend ihrer Einzelwertigkeit berücksichtigt werden.

\section{Fazit}

Das Verständnis der Pathomechanik thorakolumbaler Verletzungen und des Instabilitätsbegriffs bildet eine wichtige Grundlage in der Indikationsstellung konservativer und operativer Verfahrensweisen sowie der Zugangswege und Techniken der Stabilisation. Notwendig sind eine pathomechanisch ausgerichtete Analyse der jeweiligen Läsion hinsichtlich ligamentärer
Beteiligung und ossärer Morphologie und eine daran orientierte Verletzungsklassifikation.

Der Zustand der Instabilität im Bereich der thorakolumbalen Wirbelsäule hat fließende Übergänge zu stabilen Verletzungen und sollte in diesem Sinne nicht absolut gesehen werden. Zu differenzieren ist zwischen den verschiedenen Definitionen der Instabilität, welche nur trennscharf auf dem Gebiet der Physik vorliegt, ihrer klinischen Definition sowie den biomechanischen, neurologischen und radiologischen Bewertungen.

\section{Korrespondierender Autor PD Dr. M. Arand}

Abteilung für Unfallchirurgie, Hand- und Wiederherstellungschirurgie, Universität Ulm, Steinhövelstraße 9, 89075 Ulm E-Mail:markus.arand@medizin.uni-ulm.de

Interessenkonflikt: Der korrespondierende Autor versichert, dass keine Verbindungen mit einer Firma, deren Produkt in dem Artikel genannt ist, oder einer Firma, die ein Konkurrenzprodukt vertreibt, bestehen.

\section{Literatur}

1. Arand M, Kinzl L (2004) Verletzungen der Wirbelsäule. In: Mutschler W, Haas N (Hrsg) Praxis der Unfallchirurgie. Thieme, Stuttgart New York, S 579634

2. Blauth M (1998) Grundlagen der Wirbelsäulentraumatologie. In: Tscherne H, Blauth M (Hrsg) Wirbelsäule. Springer, Berlin Heidelberg New York, S 1 10

3. Keller TS, Hansson TH, Abram AC et al. (1989) Regional variations in the compressive properties of lumbar vertebral trabeculae: effects of disc degeneration. Spine 14: 1012

4. Louis R (1977) Les theories de l'instabilite. Rev Chir Orthop Reparatrice Appar Mot 63: 423-425

5. Magerl F, Aebi M, Gertzbein SD et al. (1994) A comprehensive classification of thoracic and lumbar injuries. Eur Spine J 3: 184-201

6. Perry O (1957) Fracture of the vertebral end-plate in the lumbar spine. Acta Orthop Scand 25S: 1

7. White AA, Panjabi MM (1990) Physical properties and functional biomechanics of the spine. In: White AA, Panjabi MM (eds) Clinical biomechanics of the spine. Lippincott, Philadelphia, pp 1-76

8. White AA, Panjabi MM (1990) The problem of clinical instability in the human spine: a systematic approach. In: White AA, Panjabi MM (eds) Clinical biomechanics of the spine. Lippincott, Philadelphia, pp 19-28 